\title{
Screening for pancreatic cancer-a compelling challenge
}

\author{
Gabriele Capurso ${ }^{1}$, Salvatore Paiella ${ }^{2}$, Massimo Falconi ${ }^{3}$ \\ ${ }^{1}$ Pancreato-Biliary Endoscopy and Endosonography Division, Pancreas Translational \& Clinical Research Center, San Raffaele Scientific Institute \\ IRCCS, Milan, Italy; ${ }^{2}$ General and Pancreatic Surgery Unit, Pancreas Institute, University of Verona, Verona, Italy; ${ }^{3}$ Pancreatic Surgery Unit, \\ Pancreas Translational \& Clinical Research Center, San Raffaele Scientific Institute IRCCS, Università Vita-Salute, Milan, Italy \\ Correspondence to: Gabriele Capurso, MD, PhD, AGAF. Pancreato-Biliary Endoscopy and Endosonography Division, Pancreas Translational \& \\ Clinical Research Center, San Raffaele Scientific Institute IRCCS, Via Olgettina 60, Milan 20132, Italy. Email: capurso.gabriele@hsr.it. \\ Comment on: Aslanian HR, Lee JH, Canto MI. AGA Clinical Practice Update on Pancreas Cancer Screening in High-Risk Individuals: Expert \\ Review. Gastroenterology 2020;159:358-62.
}

Submitted Dec 27, 2020. Accepted for publication Jan 20, 2021.

doi: $10.21037 / \mathrm{hbsn}-20-861$

View this article at: http://dx.doi.org/10.21037/hbsn-20-861

\section{Efficacy of pancreatic cancer surveillance programs}

The publication of the American Gastroenterology Association (AGA) Clinical Practice Update on Pancreas Cancer Screening in High-Risk Individuals (HRIs) underlines the increasing attention for this topic (1). Secondary prevention (surveillance) for pancreatic ductal adenocarcinoma (PDAC), however, remains a challenge with many unsolved questions (Table 1).

Revisiting the ten Wilson-Jungner criteria (2) for appraising a screening program's validity, when it comes to PDAC most have not been satisfied. Particularly, the fundamental principle that "treatment at an early stage should be of more benefit than at a later stage" has been scarcely investigated. The Johns Hopkins single-center experience reported promising efficacy results in terms of survival, with a median survival of 5.3 years [interquartile range (IQR), 1.2-11.1 years] and an outstanding $85 \%$ 3 -year survival rate (3). Notably, several studies and some meta-analyses have shown that the diagnostic yield of pre-malignant or malignant lesions in HRIs undergoing screening/surveillance is much higher than the $1.6 \%$ lifetime risk of PDAC in unselected individuals. The lifetime risk of PDAC is as high as $40-60 \%$ in PeutzJeghers syndrome (PJS) or hereditary pancreatitis (HP) patients, or in the presence of $\geq 3$ first-degree relatives (4-6). However, these reports' results do not provide evidence of survival benefits over time, leaving the issue of whether a screening/surveillance program for PDAC is effective still unsolved.

\section{Who should be screened?}

Selecting the population to be screened is crucial, and the stricter are the inclusion criteria, the higher is the diagnostic yield (7). The AGA document advises surveillance for all patients with PJS, HP and cyclin-dependent kinase inhibitor $2(C D K N 2 A)$ gene mutation, irrespective of family history and for patients with $\geq 1$ first degree relatives with PDAC with Lynch syndrome, or with mutations in BReast CAncer gene 1/2 (BRCA1, BRCA2), partner and localizer of BRCA2 (PALB2), and ataxia telangiectasia mutated (ATM) genes. Individuals with $\geq 2$ family members with PDAC of whom one first degree should also be screened. The main difference with the Cancer of the Pancreas Screening (CAPS) consortium guidelines regards the inclusion of HP, at least when associated with PRSS1 mutations. CAPS does not include these subjects. Whether patients with chronic pancreatitis $(\mathrm{CP})$, especially with early onset, associated with pathogenic mutations of serine peptidase inhibitor Kazal type 1 (SPINK1), cystic fibrosis transmembrane regulator (CFTR), chymotrypsin $\mathrm{C}$ (CTRC), carboxypeptidase $\mathrm{A} 1$ (CPA1) and carboxypeptidase B1 (CPB1) would benefit from surveillance is uncertain. As more patients with such mutations are likely to be diagnosed in the future, thanks to a more detailed investigation of causes of acute recurrent and CP, this is an important area for future research. The Italian Registry criteria for Surveillance of HRIs (7) are less rigid and allow to include all patients with a genetic cause of CP.

Also, while the AGA document underlines the importance of starting surveillance earlier in subjects with CDKN2A and PRSS1 mutations and PJS, whether an annual 
Table 1 Areas of uncertainty for pancreatic cancer surveillance

\begin{tabular}{lll}
\hline Question & Needs & Problems \\
\hline $\begin{array}{l}\text { Do surveillance protocols for PDAC } \\
\text { save lives? }\end{array}$ & $\begin{array}{l}\text { Studies on large populations of HRls } \\
\text { under active surveillance compared to other } \\
\text { HRls who do not undergo surveillance }\end{array}$ & Difficult in recruiting and having statistical power \\
Should surveillance be & $\begin{array}{l}\text { Studies developing algorithms integrating } \\
\text { personalized? }\end{array}$ & Costs of genetics, need of validation \\
Is a widespread diffusion of & genetics with environmental factors & \\
surveillance for PDAC sustainable? & $\begin{array}{l}\text { Studies on simulation of health resources } \\
\text { and costs }\end{array}$ & $\begin{array}{l}\text { Different health systems and reimbursement } \\
\text { regulations across countries }\end{array}$ \\
Is the psychological burden of & $\begin{array}{l}\text { Studies on large cohorts of HRls } \\
\text { undergoing surveillance to measure its }\end{array}$ & $\begin{array}{l}\text { Development of ad-hoc PREMs and PROMs tools } \\
\text { is needed possibly in collaboration with patients' } \\
\text { impact on quality of life }\end{array}$ \\
\hline
\end{tabular}

PDAC, pancreatic ductal adenocarcinoma; HRI, high-risk individual; PREMs, patient-reported experience measure; PROMs, patient-reported outcome measure.

examination is sufficient in these cases is also uncertain. Interval cancers have been reported in $C D K N 2 A$ mutation carriers (8), and in such cases a 6-month interval may be more appropriate. Finally, while it is plausible that other factors such as smoking (9), overweight, diabetes or diet may modify the risk of developing cancer in HRIs, this must further be ascertained.

\section{Is pancreatic cancer screening "sustainable"?}

As we head toward a personalized medicine era, more PDAC patients will receive germline testing to choose the most appropriate treatment. However, this will also lead to an enormous increase in family members considered HRIs and eligible for surveillance.

At the very least, some $5 \%$ of sporadic PDAC patients, indeed, carry germline mutations of $B R C A 1 / 2, A T M$, $P A L B 2, C D K N 2 A$, or of mismatch repair genes (10).

Thus, in the US, where almost 60,000 individuals are diagnosed with PDAC annually, at least 3,000 families would need surveillance, possibly some 10,000 new individuals per year. This estimate does not consider additional individuals meeting criteria based on family history in the absence of mutations and those with HP. As PDAC surveillance should only be performed in tertiary Centers with adequate facilities and high-volumes, we wonder whether this is a sustainable burden as part of research protocols.

\section{Psychological burden}

The need to ascertain a screening program's psychological sustainability was recognized as a relevant issue already in 1968 (1). This aspect, however, has only been marginally investigated in PDAC screening/surveillance programs. A systematic review and other studies reported low-tomoderate levels of PDAC-related distress, acceptable rates of anxiety and distress, low-to-moderate levels of PDAC perceived risk, with an acceptable psychological status $(11,12)$. However, it has been repeatedly found that subjects at a younger age may experience higher distress rates, which cannot be neglected $(12,13)$. It must be considered that the psychological burden of HRIs undergoing screening/ surveillance for PDAC may be heavily burdened by the oncological family history that had often seen the individual having played the role of caregiver of a strict relative before being a proband. Also, for some individuals (e.g., those suffering from Peutz-Jeghers or familial atypical multiple mole melanoma (FAMMM) syndromes or harboring a $B R C A 1 / 2$ mutation), this experience is exacerbated by the personal oncological history (of breast, ovarium or bowel cancers, or melanoma). The AGA guidelines do not recommend any psychological support to individuals undergoing screening/surveillance for PDAC. Instead, we believe it is advisable to include a psycho-oncologist in the team of clinicians dealing with screening/surveillance for PDAC so that any psychological distress would be promptly diagnosed and treated to obtain gains over time.

\section{Conclusions}

Prevention is likely the key issue to tackle PDAC mortality. Surveillance programs need to be refined and personalized, applying algorithms that consider genetics and other factors 
that may increase or decrease the risk of developing lesions or modify their growth rate.

The development of well-structured and widespread surveillance programs with the capability to enroll all subjects at high risk and take care of all aspects of care, including psychology, is a challenge that researchers must face worldwide and that cannot be lost.

\section{Acknowledgments}

Funding: None.

\section{Footnote}

Provenance and Peer Review: This article was commissioned by the editorial office of Hepatobiliary Surgery and Nutrition. The article did not undergo external peer review.

Conflicts of Interest: All authors have completed the ICMJE uniform disclosure form (available at https://hbsn. amegroups.com/article/view/10.21037/hbsn-20-861/coif). The authors have no conflicts of interest to declare.

Ethical Statement: The authors are accountable for all aspects of the work in ensuring that questions related to the accuracy or integrity of any part of the work are appropriately investigated and resolved.

Open Access Statement: This is an Open Access article distributed in accordance with the Creative Commons Attribution-NonCommercial-NoDerivs 4.0 International License (CC BY-NC-ND 4.0), which permits the noncommercial replication and distribution of the article with the strict proviso that no changes or edits are made and the original work is properly cited (including links to both the formal publication through the relevant DOI and the license). See: https://creativecommons.org/licenses/by-nc-nd/4.0/.

\section{References}

1. Aslanian HR, Lee JH, Canto MI. AGA Clinical Practice Update on Pancreas Cancer Screening in HighRisk Individuals: Expert Review. Gastroenterology 2020;159:358-62.

2. Wilson JMG, Jungner G. Principles and practice of screening for disease. Geneva: World Health Organization, 1968.

3. Canto MI, Almario JA, Schulick RD, et al. Risk of
Neoplastic Progression in Individuals at High Risk for Pancreatic Cancer Undergoing Long-term Surveillance. Gastroenterology 2018;155:740-751.e2.

4. Paiella S, Salvia R, De Pastena M, et al. Screening/ surveillance programs for pancreatic cancer in familial highrisk individuals: A systematic review and proportion metaanalysis of screening results. Pancreatology 2018;18:420-8.

5. Signoretti M, Bruno MJ, Zerboni G, et al. Results of surveillance in individuals at high-risk of pancreatic cancer: A systematic review and meta-analysis. United European Gastroenterol J 2018;6:489-99.

6. Pandharipande PV, Heberle C, Dowling EC, et al. Targeted screening of individuals at high risk for pancreatic cancer: results of a simulation model. Radiology 2015;275:177-87. Erratum in: Radiology. 2016 Jan;278(1):306.

7. Capurso G, Paiella S, Carrara S, et al. Italian registry of families at risk of pancreatic cancer: AISP Familial Pancreatic Cancer Study Group. Dig Liver Dis 2020;52:1126-30.

8. Ibrahim IS, Wasser MN, Wu Y, et al. High Growth Rate of Pancreatic Ductal Adenocarcinoma in CDKN2Ap16-Leiden Mutation Carriers. Cancer Prev Res (Phila) 2018;11:551-6.

9. Paiella S, Capurso G, Cavestro GM, et al. Results of FirstRound of Surveillance in Individuals at High-Risk of Pancreatic Cancer from the AISP (Italian Association for the Study of the Pancreas) Registry. Am J Gastroenterol 2019;114:665-70.

10. Shindo K, Yu J, Suenaga M, et al. Deleterious Germline Mutations in Patients With Apparently Sporadic Pancreatic Adenocarcinoma. J Clin Oncol 2017;35:3382-90.

11. Cazacu IM, Luzuriaga Chavez AA, Saftoiu A, et al. Psychological impact of pancreatic cancer screening by EUS or magnetic resonance imaging in high-risk individuals: A systematic review. Endosc Ultrasound 2019;8:17-24.

12. Paiella S, Marinelli V, Secchettin E, et al. The emotional impact of surveillance programs for pancreatic cancer on high-risk individuals: A prospective analysis. Psychooncology 2020;29:1004-11.

13. O'Neill RS, Meiser B, Emmanuel S, et al. Long-term positive psychological outcomes in an Australian pancreatic cancer screening program. Fam Cancer 2020;19:23-35.

Cite this article as: Capurso G, Paiella S, Falconi M. Screening for pancreatic cancer-a compelling challenge. HepatoBiliary Surg Nutr 2021;10(2):264-266. doi: 10.21037/hbsn-20-861 